\title{
Scheme for fast calculation of guided modes in planar optical waveguides
}

E. Jan de Jong

E. Jan de Jong, "Scheme for fast calculation of guided modes in planar optical waveguides," Proc. SPIE 1603, Education in Optics, (1 March 1992); doi: $10.1117 / 12.57889$

SPIE. Event: Education in Optics, 1991, Leningrad, Russian Federation 


\title{
Scheme for fast calculation of guided modes \\ in planar optical waveguides
}

\author{
Jan de Jong \\ Delft University of Technology \\ Department of Applied Physics \\ Optics Research Group, Delft \\ The Netherlands
}

\begin{abstract}
A new, two-step scheme for the calculation of complex propagation constants in planar multilayer waveguides is presented. In the first step, by introducing a 'Mode function', a fast determination of an approximate value of the complex propagation constant for a chosen waveguide mode is found. In the second step, in which a fast root-finding algorithm is used, the precise value of the propagation constant is calculated. The result is a significant speeding up of the computational process in comparison to traditional methods of determining complex propagation constants. Implemented in Turbo Pascal Version 5.5 on an MS-DOS AT personal computer with co-processor and $10 \mathrm{MHz}$ clock frequency, the propagation constant of a four layer channel waveguide with absorbing layers can be computed, using the effective index method, in 4 seconds after which the field profiles can be presented within a few seconds.
\end{abstract}

\section{INTRODUCTION}

For research in integrated optics, the calculation of the propagation constants of waveguide modes in both planar and channel waveguide structures is of fundamental importance. Once these are calculated, other properties, like the modal field distributions, can be obtained. In general, for structures containing absorbing dielectric layers and metals or for stacks permitting leaky waves (e.g. for prism-coupling), the propagation constant will be complex. Then, calculation of the propagation constant comprises finding the complex roots of a complex dispersion relation. This requires a two-dimensional search in the complex plane which is usually very time-consuming.

It would be useful for research as well as for educational purposes if the theoretically expected effect of a change in the waveguide structure (like number of layers, their thicknesses and refractive indices) on the propagation index and the field profile could be calculated and graphically presented within a few seconds on a small computer.

To this end a new, fast calculation scheme is developed. This is based upon thin-film matrix theory, combining the useful properties of the modal-dispersion function introduced by Chilwell and Hodgkinson ${ }^{1}$ with those of a complex mode function as introduced below.

\section{THIN-FILM MATRIX METHOD}

Consider a waveguide consisting of $\mathrm{J}$ homogeneous thin layers between two semi-infinite media, with arbitrary refractive indices. The media and their interfaces are numbered as depicted in Fig. 1 . The $\mathrm{x}$-axis of a cartesian coordinate system is chosen perpendicularly to the interfaces, while the positive z-axis is parallel to the propagation direction. The time- and $z$-dependences of monochromatic fields are given by

$$
\exp \left[i\left(k_{V} \beta-\omega t\right)\right] \text {. }
$$




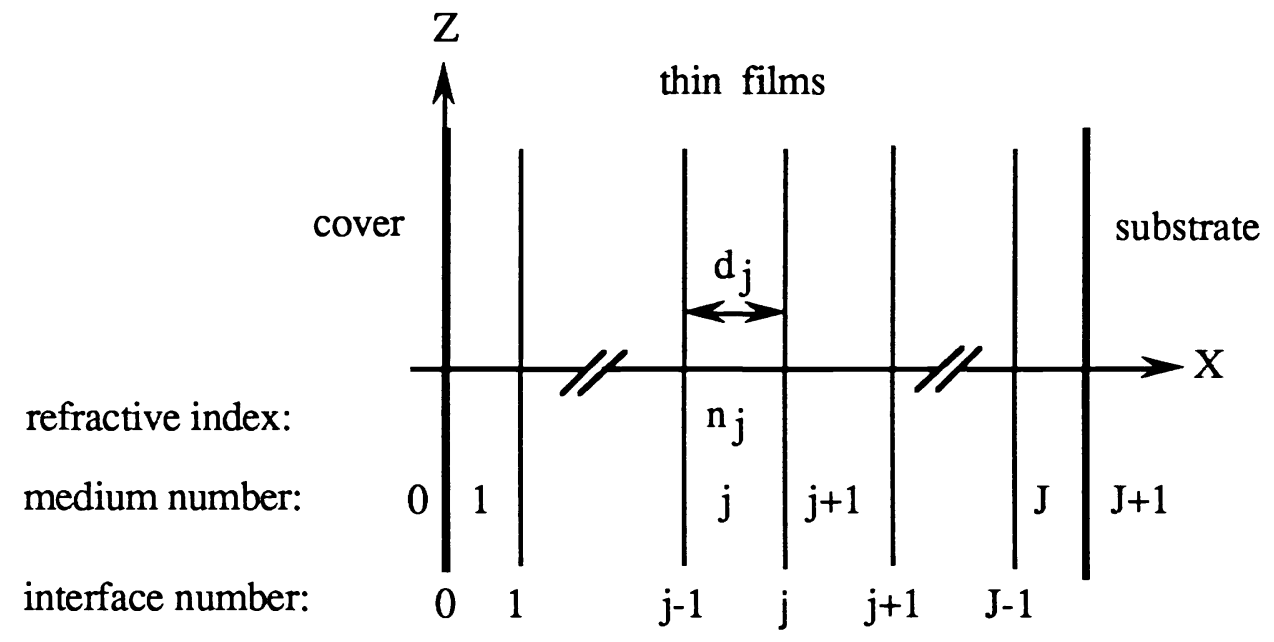

Fig.1. Multilayer stack consisting of $J$ planar films enclosed between the semi-infinite media 0 and $J+1$.

Here, $\mathrm{i}$ is the imaginary unit, $\mathrm{k}_{\mathrm{V}}$ the vacuum wave number, and $\omega$ the angular frequency. For a given waveguide mode, $\beta$ is the mode (or effective refractive) index. Defining the parameter $\alpha_{j}$ for the $j$-th layer by

$$
\alpha_{j}=\sqrt{n_{j}^{2}-\beta^{2}}
$$

we introduce the polarization-dependent quantities $\tilde{\alpha}_{j}, U$ and $V$ as shown in Table $I$. The latter two are proportional to the $y$ - and z-components of the electric or magnetic field amplitudes. According to the thin-film matrix method ${ }^{1,2}$, the field components $U$ and $V$ in the interface planes at $x_{j}$ and $x_{j-1}$ can be related ${ }^{3}$ by a unimodular matrix $\mathrm{M}_{\mathrm{j}}$, for both polarization directions:

$$
\left(\begin{array}{c}
U_{j} \\
V_{j}
\end{array}\right)=\left(\begin{array}{cc}
\cos \Phi_{j} & -\frac{\sin \Phi_{j}}{\tilde{\alpha}_{j}} \\
\tilde{\alpha}_{j} \sin \Phi_{j} & \cos \Phi_{j}
\end{array}\right)\left(\begin{array}{c}
U_{j-1} \\
V_{j-1}
\end{array}\right) .
$$

Here,

$$
\Phi_{\mathrm{j}}=\mathrm{k}_{\mathrm{v}} \mathrm{d}_{\mathrm{j}} \alpha_{\mathrm{j}}
$$

which is the $\beta$-dependent phase thickness of the $\mathrm{j}^{\text {th }}$ layer, which has a geometrical thickness $d_{j}=x_{j}-x_{j-1}$. Since $U_{j}$ and $V_{j}$ are continuous across the layer interfaces, the field components at the outermost interfaces $\mathrm{U}_{\mathrm{J}}, \mathrm{V}_{\mathrm{J}}$ and $\mathrm{U}_{0}, \mathrm{~V}_{0}$ are related by the product matrix $M$ :

$$
M=\prod_{\mathrm{j}=1}^{\mathrm{J}} \mathrm{M}_{\mathrm{j}}=\left(\begin{array}{ll}
\mathrm{m}_{11} & \mathrm{~m}_{12} \\
\mathrm{~m}_{21} & \mathrm{~m}_{22}
\end{array}\right) .
$$


Table I. Definition of the polarization dependent parameter $\tilde{\alpha}_{j}$ and the field amplitude components $U$ and $\mathrm{V}$; here, $\mathrm{Z}_{\mathrm{V}}$ is the vacuum wave impedance.

\begin{tabular}{cccc}
\hline Polarization & $\tilde{\alpha}_{j}$ & $\mathrm{U}$ & $\mathrm{V}$ \\
\hline $\mathrm{TE}$ & $\alpha_{\mathrm{j}}$ & $\mathrm{E}_{\mathrm{y}}$ & $\frac{\mathrm{H}_{\mathrm{z}}}{\mathrm{i}} \mathrm{Z}_{\mathrm{V}}$ \\
$\mathrm{TM}$ & $\frac{\alpha_{j}}{\mathrm{n}_{\mathrm{j}}^{2}}$ & $\mathrm{H}_{\mathrm{y}}$ & $\mathrm{i} \frac{\mathrm{E}_{\mathrm{Z}}}{\mathrm{Z}_{\mathrm{V}}}$ \\
\hline
\end{tabular}

The boundary conditions for the fields at the outermost interfaces are

$$
\begin{aligned}
& \mathrm{V}_{0}=-\frac{\tilde{\alpha}_{0}}{\mathrm{i}} \mathrm{U}_{0}, \\
& \mathrm{~V}_{\mathrm{J}}=\frac{\tilde{\alpha}_{\mathrm{J}+1}}{\mathrm{i}} \mathrm{U}_{\mathrm{J}},
\end{aligned}
$$

leading to the modal dispersion-relation 1,3

$$
\chi(\beta)=\frac{\tilde{\alpha}_{\mathrm{J}+1}}{\mathrm{i}}\left(\mathrm{m}_{11}-\mathrm{m}_{12} \frac{\tilde{\alpha}_{0}}{\mathrm{i}}\right)-\left(\mathrm{m}_{21}-\mathrm{m}_{22} \frac{\tilde{\alpha}_{0}}{\mathrm{i}}\right)=0 .
$$

Hence, the mode indices of the waveguide modes follow from the zeros of the modal-dispersion function $\chi(\beta)$.

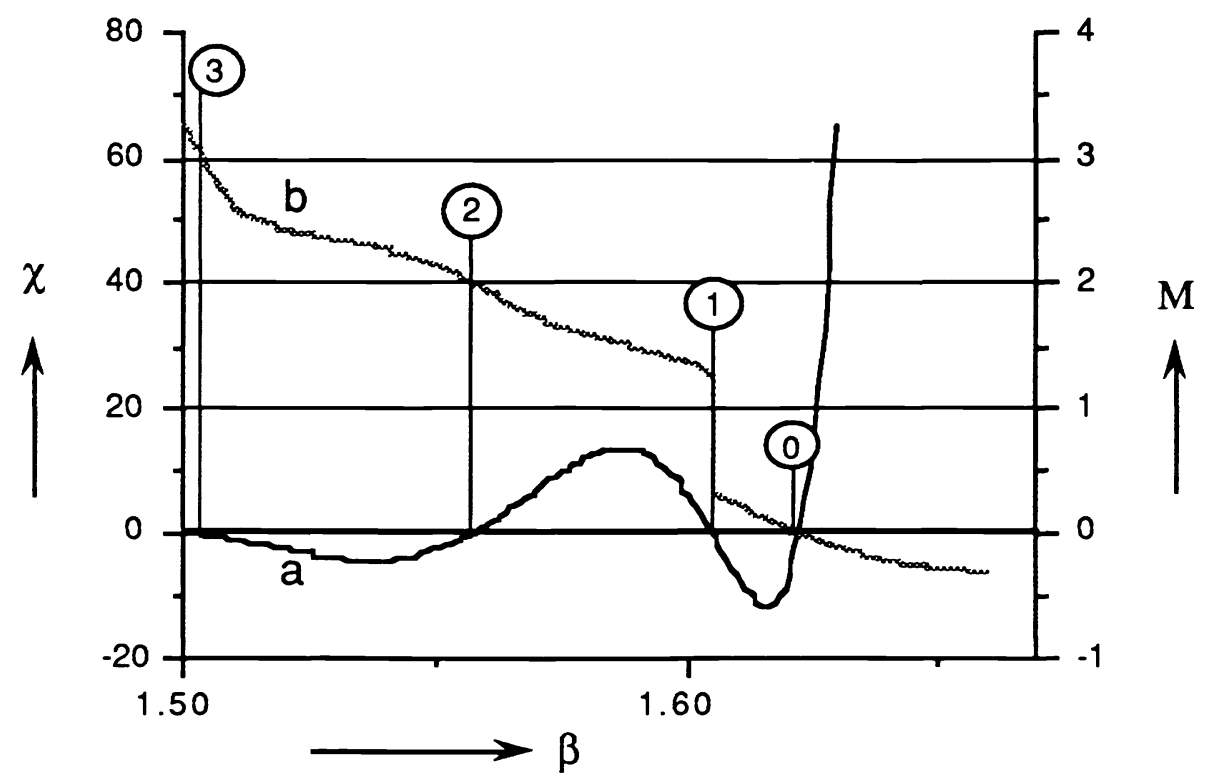

Fig. 2 a: Modal-dispersion function $\chi$ as defined by Chilwell and Hodgkinson ${ }^{1}$

b: Mode function $M$ defined here, relative to $\beta$.

Both graphs are calculated for TE polarization in the waveguide given by Table II. 
An example of this function is shown in Fig. 2, graph a, for TE polarized modes in the lossles waveguide given in Table II. Here, the vertical lines with encircled numbers denote the four possible waveguide modes. In order to calculate the mode index of order $\mathrm{m}$, all lower order modes have to be determined first starting from zero order for the zero with the highest $\beta$, see Chilwell and Hodgkinson ${ }^{1}$. For lossy modes, $\beta$ and $\chi(\beta)$ are complex. Zero determination here requires a time consuming search in the complex plane, unless an approximation can be computed more rapidly. This appears to be possible by introduction of the mode function as defined in the sequel.

Table II. Data of a four-layer waveguide as given by Chilwell and Hodgkinson 1.

\begin{tabular}{lrrrrrr}
\hline $\mathrm{j}$ & 0 & 1 & 2 & 3 & 4 & 5 \\
\hline $\mathrm{n}$ & 1.000 & 1.660 & 1.530 & 1.600 & 1.660 & 1.500 \\
$\mathrm{~d}(\mathrm{~nm})$ & & 500 & 500 & 500 & 500 & \\
\hline
\end{tabular}

vacuum wavelength: $\lambda_{\mathrm{V}}=632.8 \mathrm{~nm}$

\section{REAL MODE FUNCTION}

As long as each $n_{j}$ is real and no leaky waves are considered in a planar waveguide, $\beta$ is real, as well as $\mathrm{U}$ and $\mathrm{V}$ (apart from a constant factor) inside the waveguide. For these situations, a modal-dispersion relation has been derived ${ }^{2}$ that can provide a method for fast calculation of a waveguide mode of a specific order. A summary of the derivation is given here, which will be extended to complex values of $\beta$ in the next section.

A phase angle $\psi$ of the field $U$ inside layer $j$, apart from an integer multiple of $\pi$ is defined by

$$
\mathrm{p}\left(\begin{array}{c}
\cos \psi \\
\sin \psi
\end{array}\right)=\left(\begin{array}{c}
\mathrm{v} \\
\frac{\mathrm{v}}{v_{\mathrm{j}}}
\end{array}\right),
$$

in which the factor $\mathrm{p} \geq 0$ and

$$
v_{j}=\left|\tilde{\alpha}_{j}\right| \text {, }
$$

thus,

$$
\tan \psi=\frac{\mathrm{V}}{v_{\mathrm{j}} \mathrm{U}} .
$$

The $x$-dependence of $\psi$ inside this layer can be found after dividing $V_{j}$ and $V_{j-1}$ in Eq. (2) by $\tilde{\alpha}_{j}$. This yields 


$$
\left(\begin{array}{c}
U_{j} \\
V_{j} \\
\tilde{\alpha}_{j}
\end{array}\right)=\left(\begin{array}{cc}
\cos \Phi_{j} & -\sin \Phi_{j} \\
\sin \Phi_{j} & \cos \Phi_{j}
\end{array}\right)\left(\begin{array}{c}
U_{j-1} \\
V_{j-1} \\
\hline \tilde{\alpha}_{j}
\end{array}\right)
$$

The behaviour of $\psi$ inside a medium depends on $n_{j}$. For $n_{j}>\beta$, the matrix in Eq. (10) is a rotation matrix, hence

$$
\psi_{\mathrm{j},-}=\psi_{\mathrm{j}-1,+}+\Phi_{\mathrm{j}} \quad\left(\mathrm{n}_{\mathrm{j}}>\beta\right),
$$

where $\psi_{\mathrm{j},-}$ is the phase at $\mathrm{x}_{\mathrm{j}}-0$ and $\psi_{\mathrm{j}-1,+}$ is the phase at $\mathrm{x}_{\mathrm{j}-1}+0$, etc. Further $\mathrm{p}_{\mathrm{j},-}=\mathrm{p}_{\mathrm{j}-1,+}$. Hence, $\mathrm{U}(\mathrm{x})$ is purely cosine shaped, which is a well-known fact.

For $n_{j}<\beta$, the second term in each column matrix in Eq. (10) is multiplied by i. After substitution of Eqs. (7) and (8), this yields:

$$
\mathrm{p}_{\mathrm{j},-}\left(\begin{array}{c}
\cos \psi_{\mathrm{j},-} \\
\sin \psi_{\mathrm{j},-}
\end{array}\right)=\left(\begin{array}{cc}
\cosh \frac{\Phi_{\mathrm{j}}}{\mathrm{i}} & -\sinh \frac{\Phi_{\mathrm{j}}}{\mathrm{i}} \\
-\sinh \frac{\Phi_{\mathrm{j}}}{\mathrm{i}} & \cosh \frac{\Phi_{\mathrm{j}}}{\mathrm{i}}
\end{array}\right)\left(\begin{array}{l}
\cos \psi_{\mathrm{j}-1,+} \\
\sin \psi_{\mathrm{j}-1,+}
\end{array}\right) \mathrm{p}_{\mathrm{j}-1,+}
$$

The effect of this layer on $\psi$ is a phase difference $\Delta_{\mathrm{j}}$, defined by

$$
\psi_{\mathrm{j},-}=\psi_{\mathrm{j}-1,+}+\Delta_{\mathrm{j}} \quad\left(\mathrm{n}_{\mathrm{j}}<\beta\right) ;
$$

$\Delta_{\mathrm{j}}$ comprises hyperbolical functions of $\left|\Phi_{\mathrm{j}}\right|$, and is limited by

$$
-\frac{\pi}{2}<\Delta_{j} \leq \frac{\pi}{2}
$$

Inside this medium, $\mathrm{p}$ remains positive, but is strongly dependent on $\mathbf{x}$.

As $\psi$ depends on $n_{j}$, via $v_{j}$ (see Eq. (7)), a jump $\delta_{j}$ in $\psi$ occurs at each interface:

$$
\delta_{\mathrm{j}}=\psi_{\mathrm{j},+}-\psi_{\mathrm{j},-}
$$

Herein, $\psi_{\mathrm{j},+}$ follows from Eq. (9) with

$$
\tan \psi_{\mathrm{j},+}=\frac{v_{\mathrm{j}}}{v_{\mathrm{j}+1}} \tan \psi_{\mathrm{j},-},
$$

while

$$
-\frac{\pi}{2}<\delta_{j} \leq \frac{\pi}{2}
$$

Because the tangents of $\psi_{\mathrm{j},-}$ and $\psi_{\mathrm{j},+}$ have equal signs, these angles are in the same quadrant.

Starting from the boundary condition expressed in $\psi_{0,-}$ which follows from Eqs. (5a), (9) and (8), where $n_{0}<\beta$, we arrive at 


$$
\psi_{0,-}=-\frac{\pi}{4}
$$

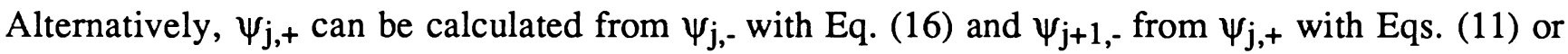
(12), using (14), resulting in $\psi_{\mathrm{J},+}$. If this satisfies the boundary condition that follows from Eqs. $(5 \mathrm{~b})$, (9) and (8):

$$
\psi_{\mathrm{J},+}=\frac{\pi}{4}+\mathrm{m} \pi \quad(\mathrm{m}=0,1,2, \ldots),
$$

the chosen $\beta$ equals the mode index of a waveguide mode. The mode order equals $\mathrm{m}$ as is proven elsewhere. ${ }^{2}$

Here, we introduce the real mode function $M(\beta)$, formally expressed in $\Phi_{j}, \delta_{j}$ and $\Delta_{j}$ as

$$
\begin{aligned}
& M(\beta)=\left(\psi_{\mathrm{J},+}-\frac{\pi}{4}\right) / \pi= \\
& \quad=\left(-\frac{\pi}{4}+\delta_{0}+\Phi_{1}+\delta_{1}+\Phi_{2}+\ldots \delta_{j-1}+\Delta_{j}+\delta_{j+1} \ldots+\delta_{J}-\frac{\pi}{4}\right) / \pi .
\end{aligned}
$$

In general, this is a monotonically decreasing function of $\beta$, because it is dominated by $\Sigma \Phi_{\mathrm{j}}$. In Fig. 2 , graph $b$, this function is depicted for the same example as in $\chi$. Thus, the mode index of the waveguide mode of order $m$ is the root of

$$
M(\beta)-m=0 \text {. }
$$

Hence, there is no need to calculate the modes of lower order in case of $m>0$. For $m>0$, this yields a substantial reduction of computing time.

\section{COMPLEX MODE FUNCTION}

Waveguide modes with complex $\beta$ can originate in waveguides with one or more lossy media or metals, or with a high index medium above a thin cover with low refractive index (prism coupling).

The thin-film matrix method is also applicable in this case. Hence, Eqs. (1) - (6) are appropriate to lossy and leaky waveguide modes.

The definition of the mode function, $M(\beta)$, can be extended to these situations.

Again, $\psi$ is defined by Eqs. (7) - (9); however, $\psi$ and $p$ are now complex. The parameter $v_{j}$ is now defined in a more general way than in Eq. (8):

$$
v_{j}=\left\{\begin{array}{ll}
\tilde{\alpha}_{j} & \left(\operatorname{Re}\left(n_{j}^{2}\right) \geq \operatorname{Re}\left(\beta^{2}\right)\right) \\
\frac{\tilde{\alpha}_{j}}{i} & \left(\operatorname{Re}\left(n_{j}^{2}\right)<\operatorname{Re}\left(\beta^{2}\right)\right)
\end{array}\right\}(j=1,2, \ldots J),
$$

and, in view of the boundary conditions in Eq. (5)

$$
v_{j}=\frac{\tilde{\alpha}_{j}}{i} \quad(j=0, J+1) .
$$

Here, care has to be taken in calculating $\alpha$. In media with $\operatorname{Re}\left(n_{j}^{2}\right)<\operatorname{Re}\left(\beta^{2}\right)$, the root with positive imaginary part has to be taken. In other layers, the root with positive real part must be taken. With these 
definitions, Eqs. (10) - (21) are applicable, provided that conditions like $n_{j}<\beta$ are interpreted as $\operatorname{Re}\left(n_{j}^{2}\right)$ $<\operatorname{Re}\left(\beta^{2}\right)$ and so on, and $\Delta_{\mathrm{j}}$ in Eq.(14) is taken as $\Delta_{\mathrm{j}}^{\mathrm{re}}\left(=\operatorname{Re}\left(\Delta_{\mathrm{j}}\right)\right)$ and $\delta_{\mathrm{j}}$ in Eq. (17) as $\delta_{\mathrm{j}}$ re

Now, $\mathrm{M}(\beta)$ can provide a means for calculation of an approximate value of the mode index of order m.

\section{FAST CALCULATION OF MODE INDEX}

For large areas of the argument, the complex function $\chi(\beta)$ is a smooth, analytical function, which is well suited for zero determinations. However, for multi mode waveguides its oscillatory character requires a two-dimensional search method in the complex plane that is very time consuming. Besides that, in order to find the zero of the order $\mathrm{m}, \beta_{\mathrm{m}}$, first all the lower order zeros have to be computed.

Here, the complex mode function $\mathrm{M}(\beta)$ can be of help to reduce the computing time, by the following observations.

1. $M(\beta)$ is much less smooth than $\chi(\beta)$ and it exhibits singularities, especially for leaky waveguides. However, for $\beta^{\mathrm{im}}=0, \mathrm{M}^{\mathrm{re}}(\beta)$ roughly decreases with $\beta^{\mathrm{re}}$. This allows of using a simple onedimensional root finding algorithm to compute the zero $\beta_{\mathrm{m} \text {,appr }}^{\mathrm{re}}$ of $\mathrm{M}^{\mathrm{re}}(\beta)-\mathrm{m}$ for $\beta^{\mathrm{im}}=0$.

2. For the most optical waveguides calculated until now, $\beta_{\mathrm{m} \text { appr }}^{\mathrm{re}}$ is a good approximation of $\beta^{\mathrm{re}}: \mid \beta_{\mathrm{m}}^{\mathrm{re}}$ $\beta_{\mathrm{m} \text { appr }}^{\mathrm{re}} \mid$ is at the highest in the order of $10^{-4}$, both for lossy and leaky modes.

3. The highest possible mode order, $\mathrm{m}_{\max }$, can practically always be found in one step as $\operatorname{Trunc}\left\{\mathrm{M}^{\mathrm{re}}(\beta)\right\}$, calculated for $\beta^{\mathrm{im}}=0$ and for $\beta^{\mathrm{re}}$ equal to the maximum of $\mathrm{n}^{\mathrm{re}}$ in cover and substrate. With $\mathrm{m}_{\max }$ calculated, a computerprogram can guard against calculating non-existent modes.

With two initial values, $\beta_{1}$ and $\beta_{2}$ which are close enough to the root, a simple and fast root finding algorithm ${ }^{4}$ can be used for computing the precise value of the root of $\chi(\beta)=0$. Good values for $\beta_{1}$ and $\beta_{2}$ appeared to be ${ }^{5}$,

$$
\begin{aligned}
& \beta_{1}=\beta_{\mathrm{m}, \text { appr }}^{\mathrm{re}}-\varepsilon^{\mathrm{re}} /\left(\mathrm{m}_{\max }+1\right) \\
& \beta_{2}=\beta_{\mathrm{m} \text {,appr }}^{\mathrm{re}}+\varepsilon^{\mathrm{re}} /\left(\mathrm{m}_{\max }+1\right)+\mathrm{i} \varepsilon^{\mathrm{im}},
\end{aligned}
$$

with $\varepsilon^{\mathrm{im}}=5 \cdot 10^{-2}$ and $\varepsilon^{\mathrm{re}}$ dependent on the maximum of the (real parts of the) refractive indices in the films, $\mathrm{n}_{\mathrm{f}, \max }$, and in cover and substrate, $\mathrm{n}_{\mathrm{cs}, \max }$ :

$$
\varepsilon^{\mathrm{re}}=0.02\left(\mathrm{n}_{\mathrm{f}, \max }^{\mathrm{re}}-\mathrm{n}_{\mathrm{cs}, \max }^{\mathrm{re}}\right) .
$$

Finally, $M(\beta)$ in this root can be computed to verify the mode order. 
Using the effective index method, the mode index at a channel waveguide can be approximated very well by repeated application of the algorithm for planar waveguides. For research and educational purposes, the algorithm described here, is implemented in Turbo Pascal 5.5 on MS-DOS personal computers. On an AT computer with $10 \mathrm{MHz}$ clock frequency, supplied with a mathematical coprocessor, the mode index of an absorbing, four layer channel waveguide with three different lateral regions, can be computed within 4 seconds. After that, in several additional seconds, the field distributions can be displayed on the screen.

\section{CONCLUSION}

For multilayer waveguides with lossy and leaky modes, a new function is introduced, the "mode function". This function assumes the value of the mode order of each waveguide mode, provided that its argument equals the mode index (= effective index of refraction). By combining the good properties of this mode function with those of the modal-dispersion function, introduced by Chilwell and Hodgkinson $^{1}$, an algorithm is developed for fast calculation of the mode index of the order of interest.

Its high calculation speed makes it feasible to implement it on a personal computer. Even the calculation time for a mode index of an absorbing channel waveguide, applying the effective index method, only is in the order of seconds on a PC.

The small response time between changing waveguide data and the computed results on a PC, makes this also a very usefull educational tool for courses on integrated optics etc.

\section{ACKNOWLEDGEMENTS}

The author has appreciated the stimulating discussions with A.G. Tijhuis, who also placed at his disposal the Fortran source of a fast root finding procedure for complex functions. He also wishes to thank M.K. Smit who gave access to his computer program WGAnal, which could be used for comparing the calculated results.

\section{REFERENCES}

1. J. Chilwell and I. Hodgkinson, "Thin-films field-transfer matrix theory of planar multilayer waveguides and reflection from prism-loaded waveguides", J. Opt.Soc.Am.A, Vol. 1, No. 7, pp. 742753, July 1984.

2. J. de Jong, "Multilayered slab waveguide design using a hybrid field vector", Appl. Opt., Vol. 28, No. 17, pp. 3567 - 3576, Sept. 1989.

3. The coordinate system is choosen as by De Jong ${ }^{2}$, while V equals his $\tilde{\mathrm{V}}$. Further, the order of calculation, here from interface $j-1$ to $j$, differs from his. These differences influence the elements of $M_{j}$ and also the function $\chi(\beta)$ and other relations.

4. We used a procedure, based on Muller's method. Author of the procedure: N.P. de Koo; English version: A.G. Tijhuis (1984); translated into Fortran: Ch. Sabharwal (1985); translated into Pascal: J. de Jong (1991).

5. Calculations are made a.o. for waveguides consisting of $\mathrm{S}_{\mathrm{i}} \mathrm{O}_{2}$ and $\mathrm{Al}_{2} \mathrm{O}_{3}$, with indices of refraction of 1.457 and 1.7 respectively, at $\lambda_{\mathrm{V}}=632.8 \mathrm{~nm}$, with and without prism. Further InGaAsP-based waveguides are calculated, with indices of refraction of 3.2887 and 3.169 for $\lambda_{\mathrm{V}}=1550 \mathrm{~nm}$. 